\title{
A Conversational Workflow Model for Chatbot
}

\author{
Francesco Colace, Francesco Pascale \\ DIIn \\ University of Salerno \\ Fisciano (SA), Italy \\ \{fcolace, fpascale\}@unisa.it
}

\author{
Antonio Ferraioli, Luca Garofalo, \\ Alfredo Troiano \\ System Management S.r.l. \\ Naples, Italy \\ \{aferraioli, lgarofalo, atroiano $\}$ \\ @ sysmanagement.it
}

\author{
Saverio Lemma, Marco Lombardi \\ SIMASLab \\ University of Salerno \\ Fisciano (SA), Italy \\ \{slemma,malombardi\}@unisa.it
}

Extended Abstract-This paper presents the realization of a prototype of a conversational workflow for a Chatbot in tires domain. The initial purpose has focused on the design of the specific model to manage communication and propose the most suitable tires for users. For this aim, it has been used the Petri Net. Finally, after the implementation of the designed model, experimental campaign was conducted in order to demonstrate its enforceability and efficiency. In research fields, the issue of Chatbot and Bot in general has been discussed for many years, although it has seen an increasingly gradual slowdown in recent years. In fact, the amount of investment by companies in trying to create a Bot as similar to an operator is growing[1][2]. The main aim of this paper is to describe a software module prototype, called workflow manager, who is responsible for the management of the flow of conversation between a Chatbot and a user, applied at a real case. Our case study concerns a Chatbot, which will serve for help the choice of tires to buy. After an analysis on the state of art, we have realized the general pattern of operation of the bot and then using the Petri Nets, we have realized the workflow model. Finally, it was conducted the experimental phase that has highlighted the strengths and weaknesses points of the Bot. In the next section, the related works are presented. The design of Workflow Manager was divided in two phases: in the first place, is provided the design of model able to explicitly describe and represent the considered knowledge domain (tires). In the second place, the purpose is to define a workflow module. Therefore, the first aim has been to build an ontology to describe the reference taxonomy. The advantage of this approach is to be able to represent concepts, properties, attributes and constraints of the one part and of the other part a reference model for the workflow[3][4]. In this work, it can be considered the child pneumatic concept. It has several children including the vehicle node, whose descendants are the cars and motorcycles node, and the node size. Each of these nodes presents, in turn, determined descendants. A choice of this type of structure has been effected because, generally, to find a specific pneumatic, must be indicated in detail the characteristics of the vehicle or the size of the eraser. Then, the ontology allows achieving a knowledge and the links between concepts (for example, we can see that we have various types of vehicles or that the concept of car is made up of the attributes of the brand, model, version and year). The next goal was to provide a reasoner that can access in a reference ontology and that it was able to generate and follow a consistent and efficient workflow, defined by a sequence of steps. The conversational module then must be able to navigate autonomously the ontology, moving through the concepts relationships. The selected model is the Petri Net because it lends well for this kind of applications. This model is composed of a set of basic objects: places, transactions and arcs, graphically represented by circles, rectangles and oriented lines. For evaluating the performance of the proposed system an experimental campaign has been developed. An implementation of the Chatbot has been developed and inserted in a Web Site of a tires seller. At the end of the chat session, an email with the suggested model tires has been sent to the potential customer. The email, showed in the store, guaranteeing a $5 \%$ discount on tires' price. In this way, it was possible to check whether the tires suggested by the system were right for the car and the needs of the customer. In two months about five hundreds potential customers (identified with the email address) used the Chatbot and 173 of them showed the email in the store and bought tires. The experimental analysis has been conducted about these 173 customers. First of all the performance of the Chatbot in providing the correct suggestions to the user has been evaluated. In particular, three different situations has been considered: Chatbot furnishes a correct suggestion, Chatbot furnishes a correct suggestion, but it does not fit with the real needs of the customer, Chatbot furnishes a wrong suggestion. The obtained results are the following Chatbot furnished the following results: Correct Suggestion: 113 $65,32 \%$, Correct Suggestion, but not suitable for the needs of the customer: 24 - 13,87\%, Wrong Suggestion: 36 - 20,81\%. Analyzing the Wrong Suggestion case, we noticed that the system fails when customer talks about a model that have various versions because it proposes tires of different dimensions. Another critical aspect occurs when the system does not understand what kind of vehicle the customer is considering. In the case of Correct Suggestion, but not suitable for the needs of the customer the main problem is in the identification of the real user needs. From the point of view of the usability a questionnaire about his/her interaction with the Chatbot was submitted to each customer. In general, they find the Chatbot easy to use and user friendly. Comparing it with other Chatbot (for example Telegram Chatbot or similar) customers says that our system is more simple and effective. In this paper, an original approach to a Chatbot has been introduced. In particular, the proposed system is based on the Petri Net formalism. A real case has been investigated developing a Chatbot, for a tires' seller. The results obtained by the experimental campaign are satisfying and show the good perspective of this kind of approach. Further developments involve the application of the proposed approach in various contexts and an improvement of the recommender approach.

\section{REFERENCES}

[1] M. Casillo, F. Colace, S. Lemma, M. Lombardi, and A. Pietrosanto, "An Ontological Approach to Digital Storytelling," In Proceedings of the The 3rd Multidisciplinary International Social Networks Conference on SocialInformatics 2016, Data Science 2016 (p. 27). ACM, 2016.

[2] F. Colace, L. Greco, S. Lemma, M. Lombardi, D. Yung, and S.K. Chang, "An Adaptive Contextual Recommender System: a Slow Intelligence Perspective," The Twenty-Seventh International Conference on Software Engineering and Knowledge Engineering (SEKE), pp. 64-71, 2015.

[3] F. Colace, M. De Santo, L. Greco, F. Amato, V. Moscato, A. Picariello, "Terminological ontology learning and population using latent dirichlet allocation," Journal of Visual Languages and Computing, 25 (6), pp. 818-826, 2014.

[4] S.K. Chang, D. Yung, F. Colace, L. Greco, S. Lemma, M. Lombardi, "An adaptive contextual recommender system: A slow intelligence perspective," Proceedings of the International Conference on Software Engineering and Knowledge Engineering, SEKE, 2015-January, pp. 64-71, 2015. 